\title{
Osteotomien - Hüftnaher Femur
}

\author{
Wolf Strecker
}

\section{Zusammenfassung}

Grundsätzlich sind 5 kongenitale und posttraumatische Zustände des proximalen Femurs durch Osteotomien operativ beeinflussbar: rezidivierende Hüftluxationen oder -subluxationen, Femurkopfnekrosen, Pseudarthrosen, Deformitäten und koxofemorale Destruktionen. Unter Berücksichtigung der individuellen Situation ist dabei in allen Fällen ein diagnostisches, indikatorisches und therapeutisches Raster zu respektieren, das sich im Wesentlichen aus folgenden Schritten aufbaut: (1.) Analyse des Problems unter Berücksichtigung von Vorgeschichte, Klinik und bildgebender Diagnostik; (2.) Indikation zur Osteotomie; (3.) zeichnerische Planung; (4.) Operationstechnik. Spezifische diagnostische, indikatorische und operationstechnische Besonderheiten der einzelnen Problemberei- che werden beschrieben. Auf Korrekturen von Deformitäten in der Frontalund Sagittalebene, von Translationen, von Längen- und Torsionsdifferenzen wird speziell eingegangen. Der klinischen, übersichtsradiografischen und computertomografischen Analyse der koxofemoralen Relation und der Beingeometrie kommt hierbei ein zentraler Stellenwert zu. Bei der Vielfalt und Komplexität kongenitaler und posttraumatischer Zustände des proximalen Femurs kann nur eine perfekte maßgeschneiderte Lösung die Antwort auf ein ganz individuelles Problem sein.

\section{Proximal Femur Osteotomy}

Basically, five post-traumatic conditions of the proximal femur can be improved by corrective osteotomies: recurrent luxations and subluxations of the hip joint; necrosis of the femoral head; non-unions; deformities; coxofemoral distraction. Depending on the individual situation, the following procedure can be recommended, including diagnosis of deformities as well as indications and therapy for corrective osteotomies: (i) analysis of the problem from the viewpoint of history, clinical findings and imaging techniques; (ii) indication for corrective osteotomy; (iii) graphic planning; (iv) operation technique. Particular features of posttraumatic and congenital conditions with regard to diagnosis, indication and surgical technique are indicated. Only perfectly tailored concepts can be expected to respond to the individual situation of an individual patient and reflect the variety and complexity of congenital and post-traumatic conditions of the proximal femur.

\section{Einleitung \\ Osteotomien am proximalen Femur, also dem Segment 31 der AO-Klassifikation [5] entsprechend, können bei folgenden Pathologien indiziert sein: \\ - rezidivierende Hüftluxationen oder -subluxationen, \\ - Femurkopfnekrosen, \\ - Pseudarthrosen, \\ - Deformitäten, \\ - koxofemorale Destruktion.}

Diese unterschiedlichen Pathologien erfordern teilweise völlig verschiedenartige therapeutische Strategien und operative Umsetzungen entsprechend der differenten Zielvorgaben:

OP-JOURNAL 2011; 27: 240-247

(c) Georg Thieme Verlag KG Stuttgart · New York DOI http://dx.doi.org/10.1055/s-0031-1280446
- störungsfreie Artikulation,

- Druckentlastung,

- Verbesserung und Verstärkung der Krafteinleitung,

- Optimierung der koxofemoralen Balance, Funktion und Anatomie,

- Sicherstellung einer Hüftgelenksfunktion.

Ungeachtet der unterschiedlichen zugrunde liegenden Pathologien ist für das Verständnis der individuellen Problematik ein standardisiertes diagnostisches, indikatorisches und therapeutisches Vorgehen zu empfehlen. Dieses baut sich im Wesentlichen aus folgenden Schritten auf:

1. Analyse des Problems unter Berücksichtigung von Vorgeschichte, Symptomatik, klinischer Untersuchung und bildgebender Diagnostik.

2. Indikation zur Osteotomie unter Würdigung indikatorischer Grenzen, loka- ler und patientenbezogener Kontraindikationen und Alternativverfahren.

3. Zeichnerische Planung.

4. Operationstechnik.

\section{Rezidivierende Hüftluxationen und -subluxationen}

Traumatische Hüftluxationen ohne knöcherne Beteiligung oder dysplastische Komponente sind äußerst selten. Bei dieser Konstellation lassen sich posttraumatische Rezidivluxationen oder -subluxationen nur durch eine Zerreißung des Kapsel-Labrum-Komplexes erklären. Bei ventralen Läsionen werden hierbei Luxationen durch Außendrehung des Beines provoziert, bei dorsalen Läsionen prinzipiell durch Innendrehung.

Kapsel-Labrum-Rekonstruktionen nach älteren Zerreißungen erscheinen bei hohem operationstechnischen Aufwand und Risiko wenig Erfolg versprechend, 


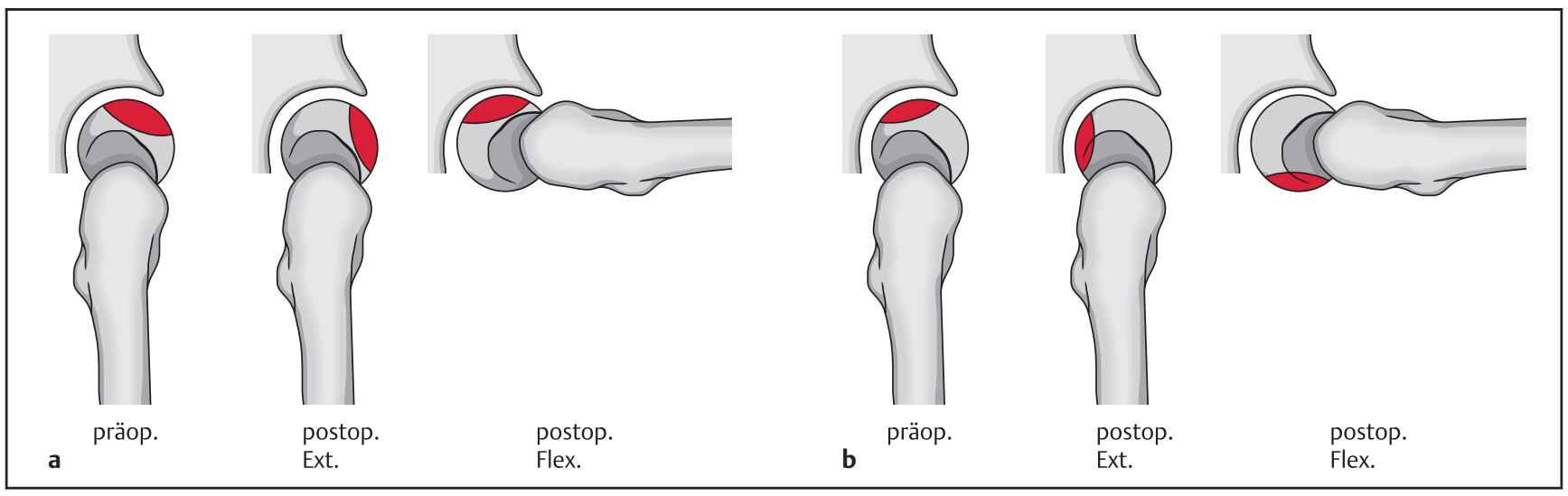

Abb. $1 \mathbf{a}$ und $\mathbf{b}$ a Anteriore Drehosteotomie nach Sugioka et al. [12]. Eine Entlastung der apikalen Femurkopfnekrose wird nur in Extension erreicht. b Posteriore Drehosteotomie nach Kempf et al. [3]. Ein Entlastungseffekt tritt sowohl in Extension als auch in Flexion ein.

sodass bei jüngeren Patienten mit adäquater Knorpelqualität eine subtrochantäre Torsionskorrektur diskutiert werden kann. Ziel dieser Torsionskorrektur ist die Vermeidung einer luxationsauslösenden Grenzrotation. Bei ventralem Kapsel-Labrum-Defekt und vorderer Hüftluxation, provoziert durch verstärkte Außendrehung des Beines, bewirkt eine subtrochantäre Außentorsionsosteotomie eine entsprechende Innentorsion von Schenkelhals und Hüftkopf mit konsekutiver Entlastung der ventralen Anteile des Hüftgelenks. Prinzipiell entspricht dieses Vorgehen der subkapitalen Drehosteotomie des Oberarms nach Weber bei rezidivierenden Schulterluxationen [9]. Grundsätzlich muss hierbei allerdings ein freier rotatorischer Nulldurchgang erhalten bleiben. Frischere Kapsel-Labrum-Läsionen sollten hingegen rekonstruiert werden, sei es arthroskopisch oder offen.

\section{Femurkopfnekrosen}

Posttraumatische Femurkopfnekrosen treten nach inadäquater axialer (Stauchung) oder tangentialer (Scherung) Krafteinwirkung auf sowie durch traumatische oder iatrogene Störungen der lokalen Durchblutung. Hauptursachen sind demnach Hüftluxationen, meist in Kombination mit Azetabulumfrakturen sowie auch (späte!) Repositionsmanöver und invasive operative Rekonstruktionen. Kombinationsverletzungen mit Kalottenfrakturen des Hüftkopfs können in einzelnen Fällen vorkommen. Aufgrund des Verletzungsmechanismus dürften isolierte traumatisch bedingte Hüftkopfnekrosen ohne Schädigung des korrespondierenden azetabulären Knorpels eher selten sein. Ein weiteres Problem stellt die meist späte bildgebende De- maskierung einer derartigen Hüftkopfnekrose dar.

Das Ausmaß der avitalen Zone wird gelegentlich erst 2-3 Jahre nach dem initialen Trauma radiografisch sichtbar. Bei entsprechendem klinischem Verdacht ist daher immer eine Magnetresonanztomografie (MRT) frühzeitig zu fordern.

Als Ursachen nicht traumatischer Femurkopfnekrosen mit entsprechenden fokalen Ischämien werden u.a. Äthylismus, Zigarettenrauchen, Immunsuppressiva und Glukokortikoide angeschuldigt.

Lediglich bei isolierten Hüftkopfnekrosen, deren Tiefenausdehnung den Kopfdurchmesser nicht um mehr als ein Drittel überschreitet, kann bei jüngeren Patienten eine Osteotomie mit dem Ziel einer lokalen Druckentlastung avisiert werden. Inwieweit zusätzliche Bohrungen mit Perforation einer ossären Sklerosezone einer Verbesserung der lokalen Biologie dienen, bleibt spekulativ. Ebenso wenig liegen verlässliche Langzeitergebnisse alternativer Maßnahmen vor, wie das lokale Einbringen von autogener Spongiosa oder mikrovaskulär gestieltem Knochen.

Art und Ausmaß von druckentlastenden Osteotomien orientieren sich letztlich an Lokalisation und Ausdehnung der $\mathrm{Ne}-$ krosezone. Liegen intakte anteriore oder posteriore Kopfanteile vor, können diese durch eine Drehosteotomie des Schenkelhalses in die Hauptbelastungszone gebracht werden. Bei der anterioren Drehosteotomie nach Sugioka et al. [12] wird die apikale Nekrosezone nach ventral gedreht, bei der posterioren Drehosteotomie nach Kempf et al. [3] nach dorsal (Abb. 1).
Als Osteosyntheseverfahren eignen sich Winkelplatten und Schraubenosteosynthesen. Aufgrund der vorbestehenden gestörten Vaskularität wird vor größervolumigen Implantaten im Bereich von Schenkelhals und Femurkopf gewarnt.

Insgesamt sollte die Indikation zur Entlastungsosteotomie bei Hüftkopfnekrosen selbst bei jüngeren Patienten sehr streng gestellt werden. Voraussetzung ist immer eine ausreichende Motivation und Compliance der betroffenen Patienten. Selbstverständlich muss die schädigende Noxe ausgeschaltet werden. Die Indikation zur Entlastungsosteotomie wird weiterhin relativiert durch neuere, vielversprechende Entwicklungen auf dem Gebiet der Endoprothetik.

\section{Pseudarthrosen}

Nach Schenkelhalsfrakturen liegt die Pseudarthrosenrate auch bei adäquater operativer Versorgung in einer Größenordnung von $10-25 \%$ und damit höher als nach Frakturen anderweitiger anatomischer Lokalisation. Hauptursache, neben traumatisch und/oder iatrogen gestörter Vaskularität, sind biomechanische Faktoren.

Pauwels hat 1935 als Erster auf die Zusammenhänge zwischen Neigungswinkel der Bruchflächen und der knöchernen Heilung hingewiesen [6]. „Je stärker die Bruchfläche geneigt ist, umso größer ist die Schubspannung, welche das Gewebe im Bruchspalt dehnt und dadurch zur Entstehung einer bindegewebigen Narbe führt." Diese Erkenntnisse führten zum einen zur bewährten Einteilung der Schenkelhalsbrüche in die 3 Grade nach Pauwels, zum anderen zu den theoretischen und praktischen Grundlagen der valgisierenden Umlage- 


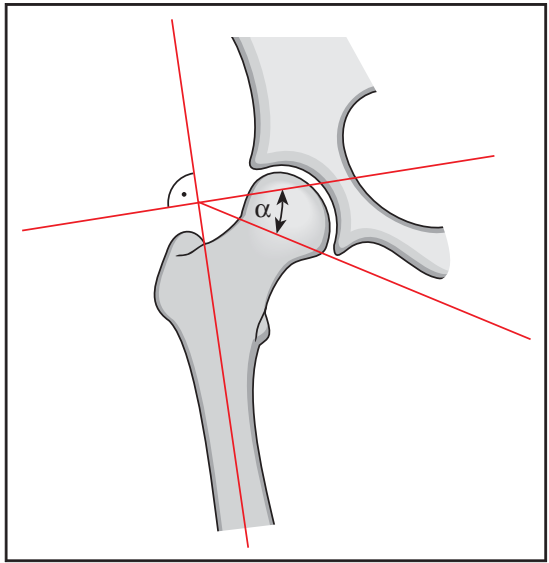

Abb. 2 Die knöcherne Heilung einer Schenkelhalsfraktur oder -pseudarthrose ist bei einem Winkel $\alpha<30^{\circ}$ zu erwarten. $\alpha$ wird definiert durch die Ebene der Fraktur bzw. Pseudarthrose und dem Lot zur anatomischen Femurachse.

rungsosteotomie. Ziel der Valgisationsosteotomie bei Schenkelhalsfrakturen und -pseudarthrosen ist die möglichst rechtwinklige Ausrichtung des Frakturbzw. Pseudarthrosespalts zu den lokal einwirkenden Druckkräften (Abb. 2).

Planerische und operationstechnische Einzelheiten werden von Maurice E. Müller im Manual der Osteosynthese ausführlich beschrieben [4]. Seine grundlegenden Erkenntnisse über die knöcherne Heilung von Pseudarthrosen unter Kompression wurden nahezu zeitgleich von G.A. Ilizarov bestätigt und zum Prinzip erklärt [2].

Die Valgisationsosteotomie (Abb. 3) ist indiziert bei (biologisch) jüngeren $\mathrm{Pa}$ tienten ohne Zeichen einer Koxarthrose oder höhergradigen Femurkopfnekrose (Abb.4a,b). Bei Kindern und Jugendlichen ist die Operationsindikation großzügig zu stellen. Selbst bei Vorliegen von Hüftkopfnekrosen wurden Revaskularisierungen nach Umlagerungsosteotomien bei einzelnen jungen Patienten beschrieben. In jedem Fall sind jedoch die Unwägbarkeiten einer derartigen operativen Korrektur, insbesondere im Hinblick auf das Schicksal des Hüftkopfs, explizit anzusprechen und schriftlich zu fixieren. Alternativ sind, unter Berücksichtigung der individuellen Situation, Vor- und Nachteile alloarthroplastischer Maßnahmen zu diskutieren.

Bei der zeichnerischen Planung von intertrochantären Valgisationsosteotomien wird ein Winkel $\alpha<30^{\circ}$ angestrebt (Abb. 2). Bei der Planung müssen selbstverständlich auch die entsprechenden

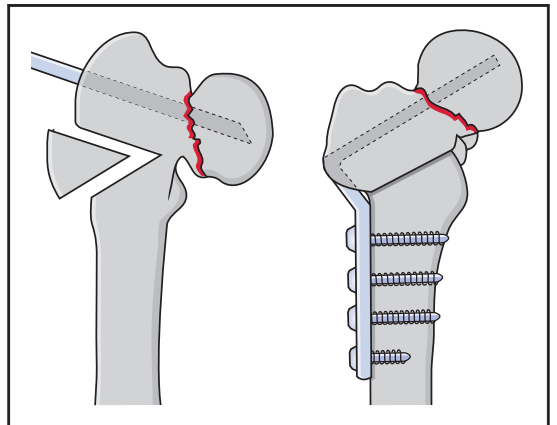

Abb. 3 Intertrochantäre Valgisationsosteotomie um $52^{\circ}$ und Osteosynthese mit einer gekröpften $120^{\circ}$-Winkelplatte. Damit wird ein Winkel $\alpha$ von $30^{\circ}$ erreicht.

Änderungen der gesamten Hüft- und Beingeometrie mit berücksichtigt werden. Dies schließt die frontalen Achsverhältnisse ebenso ein wie Veränderungen der Beinlänge und des femoropelvinen Offsets. Vorübergehende Verlängerungen können hierbei bewusst in Kauf genommen werden. Allerdings nur unter der Voraussetzung, nach Ausheilung der Pseudarthrose eine anschließende Reva- risierung und Wiederherstellung der anatomischen Situation mit einem physiologischen CCD-Winkel anzuschließen. Eine derartige Strategie muss mit dem Patienten eingehend besprochen werden. Weniger ausgeprägte Valgisationen mit einem CCD-Winkel unter $140^{\circ}$ können im Einzelfall nach Ausheilung der Pseudarthrose und entsprechender Beschwerdefreiheit des Patienten ggf. auch belassen werden. Die jeweilige Vorgehensweise ist auf den Einzelfall abzustimmen und mit dem Patienten nach gewissenhafter Nutzen-Risiko-Abwägung gemeinsam zu entscheiden.

\section{Deformitäten}

Es gibt eine Vielzahl von Ursachen von Deformitäten des proximalen Femurs. Zweifelsohne am häufigsten sind kongenitale und posttraumatische Fehlstellungen, die im Folgenden etwas näher beleuchtet werden sollen. Dennoch sei auf weitere Ursachen verwiesen, wie etwa auf longitudinale Reduktionsfehlbildungen, etwa beim proximalen foka-

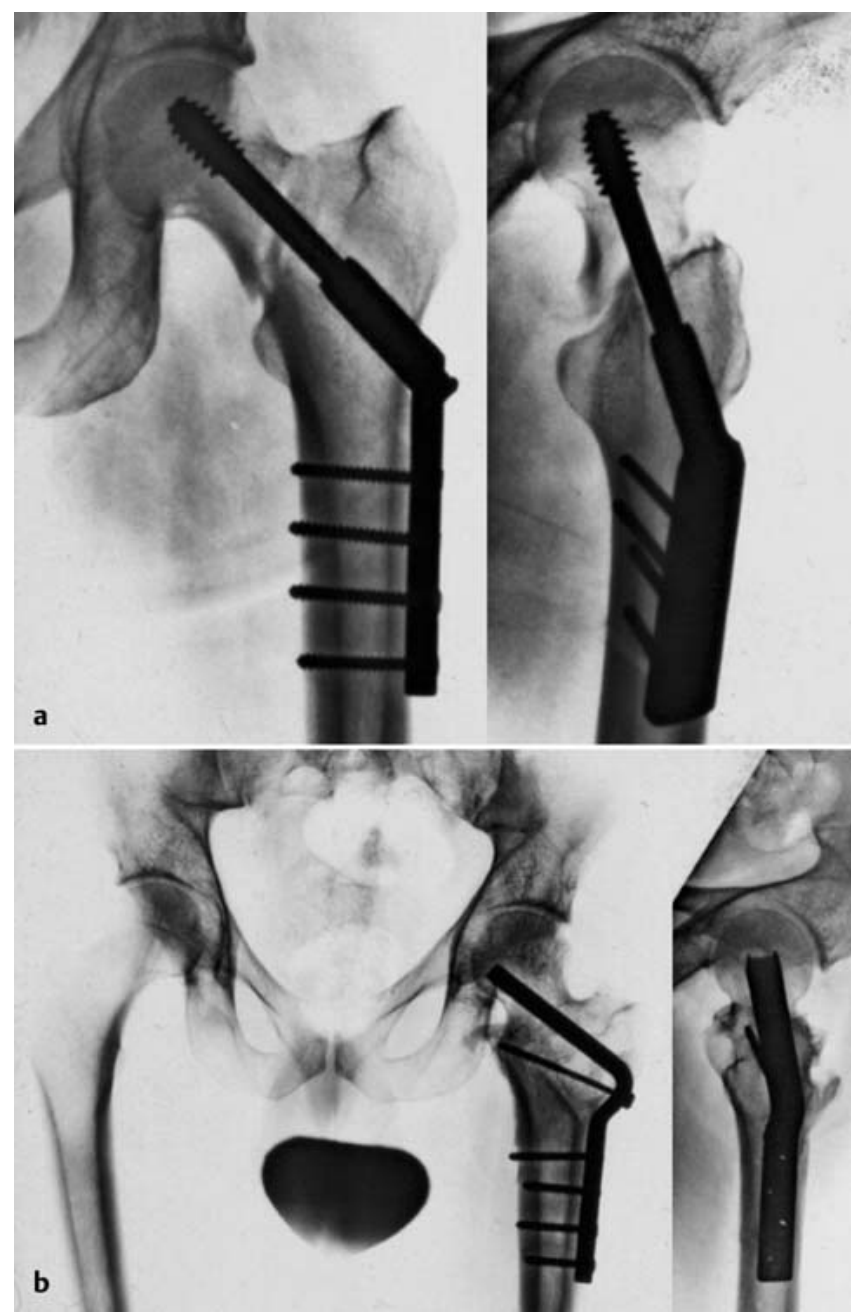

Abb. $4 \mathrm{a}$ und $\mathbf{b}$

29-jähriger Patient: a Ausbleibende Knochenheilung 5 Monate nach Schenkelhalsfraktur vom Typ Pauwels III und Osteosynthese mittels DHS. b Knöcherne Heilung der Pseudarthrose nach intertrochantärer Valgisationsosteotomie und Osteosynthese mit gekröpfter $120^{\circ}$ Winkelplatte (mit freundlicher Genehmigung von Springer Science + Business Media: Strecker W. Analyse und Korrektur von Deformitäten der oberen und unteren Extremität. In: Weigel B, Nerlich ML, Hrsg. Praxisbuch Unfallchirurgie 2011: 9571012). 
len Femurdefekt, auf diverse Formen von Osteodystrophien, metabolische, endokrine und kinetische Störungen der Knochenreifung, wie etwa bei Osteogenesis imperfecta, Achondroplasie, renaler Osteodystrophie, etc. Hinzu kommen tumorbedingte oder -assoziierte Deformitäten sowie infektiöse bzw. postinfektiöse koxofemorale Dysbalancen.

Eindimensionale Deformitäten sind selten. Die meisten typischen kongenitalen Deformitäten wie Coxa valga antetorta oder Coxa vara retrotorta sind zumindest 2-dimensional, also hier die Frontalebene und die Torsion betreffend. Posttraumatische Deformitäten betreffen zudem häufig weitere Achsabweichungen, etwa in der Sagittal- und Horizontalebene, und sind zudem nicht selten mit Längendifferenzen im Seitenvergleich vergesellschaftet. Für die planerische Analyse jeder einzelnen Deformität empfiehlt sich indessen ein systematisches Vorgehen, sowohl die klinische Untersuchung als auch die bildgebende Diagnostik betreffend. Hierbei sollte jede einzelne räumliche Dimension gewissenhaft abgefragt werden, beginnend mit der Frontalebene, gefolgt von der Sagittal- und Horizontalebene und schlussendlich der jeweiligen Längen- und Torsionsverhältnisse [8]. Am proximalen Femur von besonderer Bedeutung ist hierbei die koxofemorale Relation, die individuelle Beckenanatomie mit einschließend. Hierbei ist die Hüftgelenksbeweglichkeit in allen Richtungen des Raumes gewissenhaft zu untersuchen, insbesondere auch der Rotationsumfang nach innen und außen in $90^{\circ}$ Hüftflexion und in $0^{\circ}$ Hüftextension. Wichtige Informationen zum Verständnis der koxofemoralen Geometrie liefert die Computertomografie, ggf. auch in 3-D-Rekonstruktionen.

Im Folgenden werden die einzelnen räumlichen Dimensionen am proximalen Femur dargestellt.

\section{Frontale Achsabweichungen}

Nach valgisierender Umlagerungsosteotomie bei Schenkelhalspseudarthrose und knöcherner Ausheilung kann ein zu steiler CCD-Winkel resultieren. Übertrifft der postoperative CCD-Winkel die gesunde Gegenseite um mehr als 10$15^{\circ}$, empfiehlt sich bei jüngeren Patienten die Revarisierung. Ansonsten stellen posttraumatische Valgusdeformitäten Raritäten dar, die zudem selten korrekturbedürftig sind. Sehr viel häufiger sind kongenitale Coxa valga, meist in Verbin-

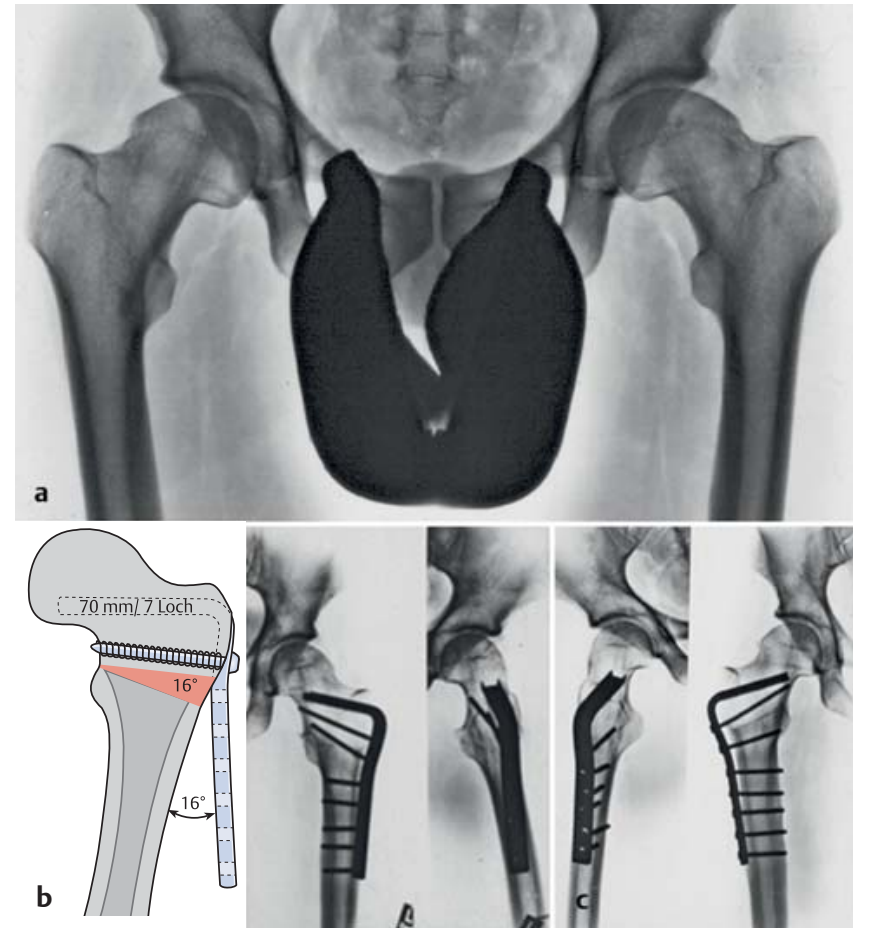

Abb. 5 a bis c 27-jähriger Patient: a Kongenitale Coxa vara retrotorta mit absolut erhöhten Außentorsionen beidseits femoral und fehlendem rotatorischem Nulldurchgang. b Intertrochantäre Valgisations $\left(16^{\circ}\right)$ Innentorsions $\left(15^{\circ}\right)$-Osteotomie mit Osteosynthese durch $95^{\circ}$-Kondylenplatte links. c 7 Monate später analoge Korrektur rechts. Radiografisch deutliche Harmonisierung der koxofemoralen Kongruenz. Dementsprechend erhebliche Verbesserung der Hüftgelenksbeweglichkeit in allen Richtungen, insbesondere der Abduktion und des Rotationsumfangs (mit freundlicher Genehmigung von Springer Science + Business Media: Strecker W. Analyse und Korrektur von Deformitäten der oberen und unteren Extremität. In: Weigel B, Nerlich ML, Hrsg. Praxisbuch Unfallchirurgie 2011: 957-1012).

dung mit einer erhöhten femoralen Innentorsion, demnach also Coxa valga antetorta. Bei CCD-Winkeln von mehr als 140-145 kann hier eine intertrochantäre Varisierung, ggf. in Kombination mit einer Torsionskorrektur, indiziert sein. Ziel ist zum einen eine Verminderung des koxofemoralen Gelenkbinnendrucks durch die Varisierung und zum anderen grundsätzlich ein freier rotatorischer Nulldurchgang im Hüftgelenk von wenigstens jeweils $15^{\circ}$ Innen- und Außenrotation. Intertrochantäre Valgisierungen gehen tendenziell mit einer Verlängerung des Beines einher, intertrochantäre Varisierungen mit einer Verkürzung. Dies ist planerisch und operativ zu berücksichtigen.

Die Operationsplanung beinhaltet nicht nur die exakte Osteotomiehöhe und den Korrekturwinkel, sondern auch die Lage des gewünschten Implantats. Hierbei empfehlen sich die Klingenplatten der AO [4]. Bei der operativen Umsetzung der Planung sollte zunächst das Klingenlager vorbereitet werden. Nach
Festlegen der intertrochantären Osteotomiehöhe mit einem Kirschner-Draht empfiehlt es sich, zur Torsionskontrolle proximal und distal der Osteotomie je eine Schanz-Schraube von $5 \mathrm{~mm}$ Durchmesser einzudrehen. Diese SchanzSchrauben sollten in axialer Aufsicht parallel liegen. In Abhängigkeit von den Längenverhältnissen wird die Knochendurchtrennung als Halbschaft- oder Ganzschaftosteotomie geplant.

Posttraumatische Varusdeformitäten sind häufiger. Ursache ist meist eine ungenügende knöcherne Abstützung medial oder eine mangelhafte Reposition und fehlerhafte Implantatlage nach Frakturen des koxalen Femurendes. Im Rahmen der zunehmenden Varisierung verkürzt sich das Femur und es entsteht in Abhängigkeit vom Apex der Deformität u.U. eine pelvitrochantäre muskuloligamentäre Insuffizienz. Die Indikation zur Korrektur varischer Deformitäten orientiert sich am Beschwerdebild des Patienten mit seinen funktionellen Problemen. Im Einzelnen gehen folgende Aspekte in die Betrachtung ein: 


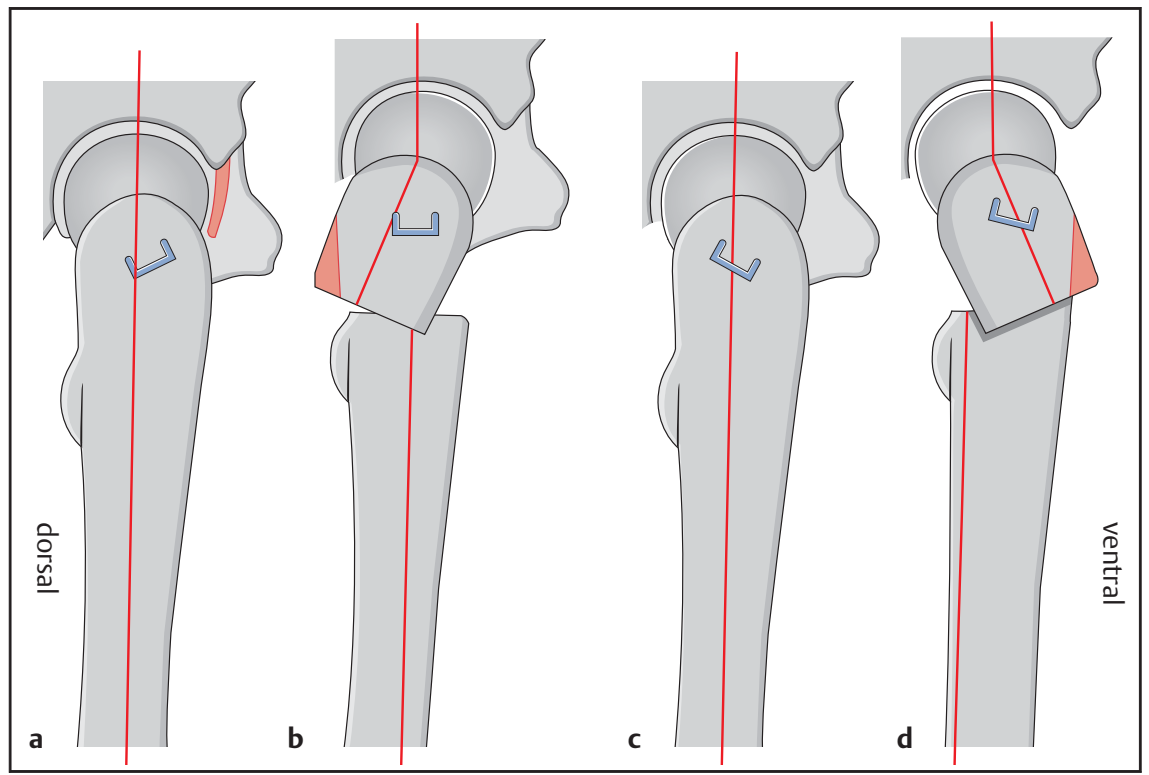

Abb. $\mathbf{6 a}$ bis d a Bei geplanter Flexionsosteotomie wird zunächst die ventrale Hüftgelenkskapsel eingekerbt oder reseziert, um eine adäquate Hyperextension im Gelenk zu erlauben. Dann Vorbereiten des Klingensitzes für die Winkelplatte im gewünschten sagittalen Korrekturwinkel. b Intertrochantäre Querosteotomie und Eintreiben der Winkelplatte mit Komplettieren der Osteosynthese unter interfragmentärer Kompression. Knöcherne Überstände dorsal können abschließend abgetragen und als Spongiosa ventral angelagert werden. c, d Analoges Vorgehen bei Streckdefizit im Hüftgelenk und geplanter intertrochantärer Extensionsosteotomie. Hierbei muss die dorsale Hüftgelenkskapsel nicht inzidiert werden.

- Trendelenburg-Hinken,

- Einschränkungen des Bewegungsumfangs,

- laterale Ausladung des Trochanter major,

- Verlauf der mechanischen Tragachse mit potenzieller Fehlbelastung von Knie- und Sprunggelenken,

- Ausmaß der begleitenden Oberschenkelverkürzung,

- Kopf-Pfannen-Relation des Hüftgelenks.
Kongenitale Coxa vara manifestieren sich häufig als Coxa vara retrotorta und scheinen bei Männern häufiger aufzutreten als bei Frauen (Abb. 5). Die Beweglichkeit der Hüftgelenke ist bez. Abduktion und Innenrotation oft erheblich eingeschränkt. Adduktionsaufnahmen in neutraler Hüftrotation können Hinweise auf das Ausmaß einer möglichen Valgisierung geben. Bei diesen Aufnahmen stellt sich die koxofemorale Kongruenz nach einer entsprechenden Korrektur dar. Analog zu den periazetabulären Osteotomien sollte auch hier der Arthrosegrad nach Kellgren nicht über 2 liegen. Entsprechende Korrekturen werden zudem auf Patienten mit einem biologischen Alter von weniger als 50 Jahren beschränkt. Differenzialdiagnostisch und -indikatorisch ist ein isoliertes koxofemorales Impingement abzugrenzen [1] Hierbei sind zusätzliche kongenitale Torsionsdeformitäten auszuschließen [11].

\section{Sagittale Achsdeformitäten}

Von klinischer Relevanz sind kongenitale oder posttraumatische Streckdefizite im Hüftgelenk, die von den benachbarten Gelenken, hier also insbesondere von Lendenwirbelsäule und Kniegelenk, kompensiert werden müssen. Diese funktionelle Beeinträchtigung der benachbarten Gliederkette geht mit einer Verkippung des Beckens sowie einer lumbalen Lordosierung einher. Streckdefizite des Hüftgelenks von mehr als $10^{\circ}$ können daher eine Korrekturosteotomie indizieren. Beugedefizite sind von geringerer Relevanz und bedürfen nur ausnahmsweise einer Korrektur. Weitere Indikationen sind segmentale Hüftkopfnekrosen und fokale Arthrosezonen bei jüngeren Menschen. Durch die intertrochantäre Extensions- oder Flexionsosteotomie (Abb. 6) soll der nekrotische oder arthrotische Gelenkabschnitt aus der Hauptbelastungszone des Hüftgelenks herausgedreht werden, analog zu den Drehosteotomien nach Kempf [3] und Sugioka [12]. Bei mehrdimensionalen Deformitäten lassen sich sagittale Korrekturen meist gut integrieren, wie etwa bei que-
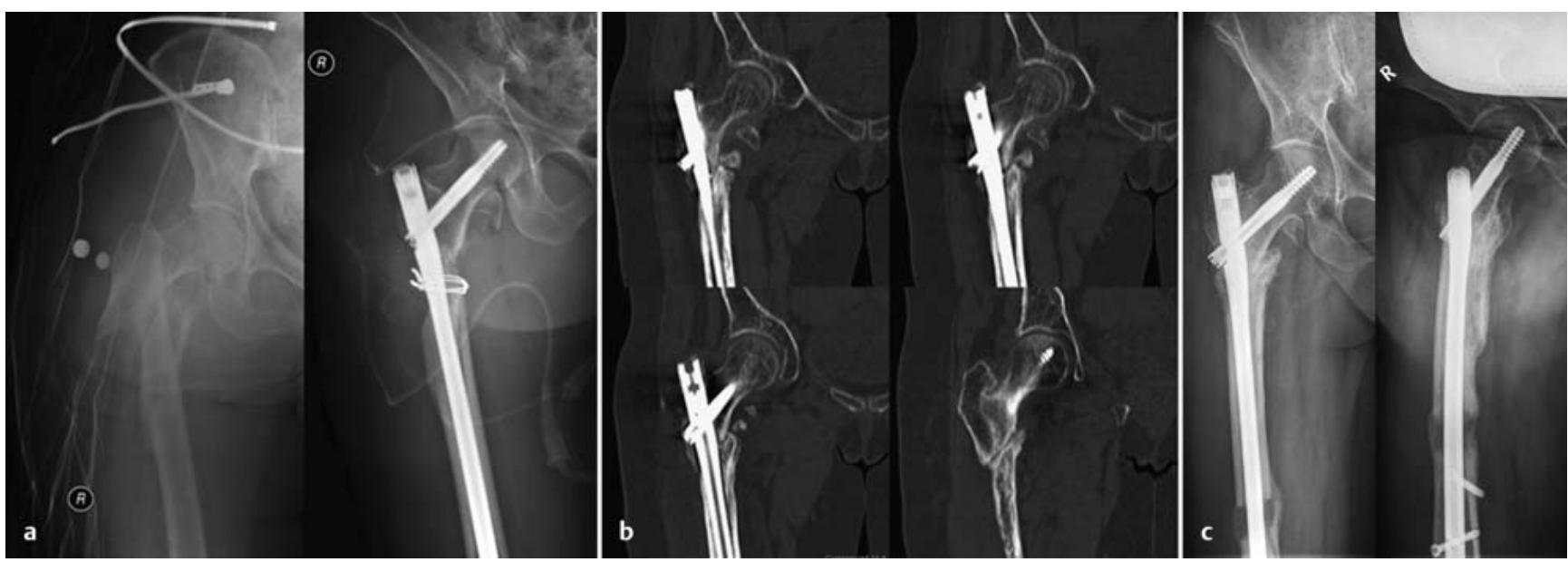

Abb. 7a bis c 51-jährige Patientin mit a proximaler Femurmehrfragmentfraktur rechts vom Typ AO 31-A2.3. Primär insuffiziente Reposition, dennoch Retention mit langem $y$-Nagel. b Es verbleibt eine subtrochantäre Pseudarthrose sowie zusätzlich eine Verkürzungs $(1,8 \mathrm{~cm})$-Außentorsions $\left(25^{\circ}\right)$-Deformität. c Regelrechte Beingeometrie und Ausheilung der Pseudarthrose nach akuter diaphysärer Innentorsions $\left(25^{\circ}\right)$-Osteoklasie und Anlage eines unilateralen Distraktionsfixateurs zur kontinuierlichen Verlängerung und gleichzeitigen Kompression der Pseudarthrose durch Segmenttransport. Hier Röntgenkontrolle nach distaler statischer Verriegelung, zusätzlicher Pollerschraube und Entfernung des Distraktionsfixateurs. 


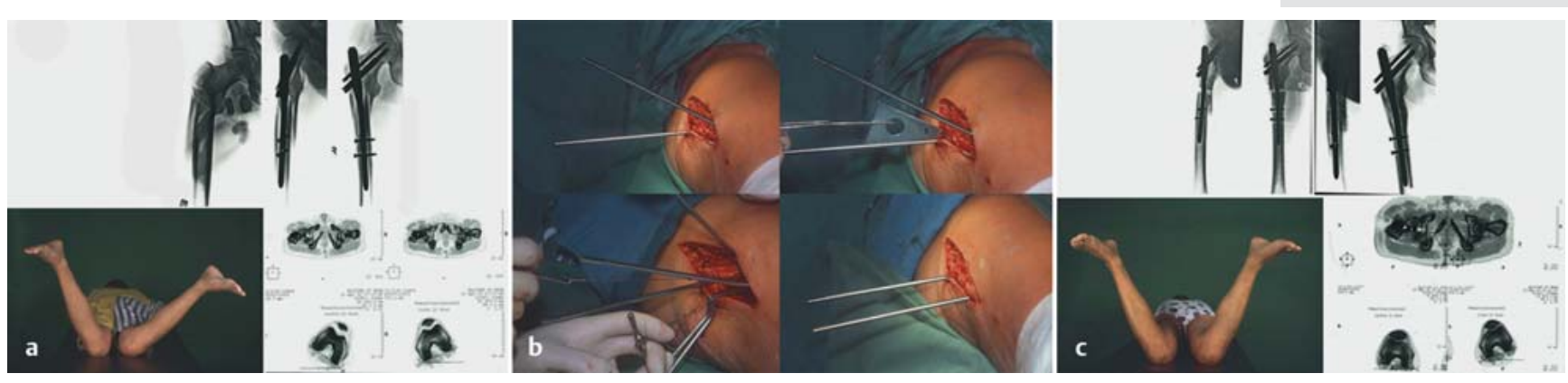

Abb. 8 a bis c 55 -jähriger Patient mit a posttraumatischer Verkürzungs $(1,4 \mathrm{~cm})$-Innentorsions $\left(41^{\circ}\right)$-Deformität rechts femoral nach Osteosynthese einer per- und subtrochantären Mehrfragmentfraktur. b Indikation zur Verlängerungs $(1,2 \mathrm{~cm})$-Außentorsions $\left(40^{\circ}\right)$-Osteotomie subtrochantär gegeben. Proximal und distal der geplanten Gigli-Osteotomie Eindrehen von 5-mm-Schanz-Schrauben als Zeiger für die akute Außentorsionskorrektur. c Gleichzeitig akute Verlängerung mit Anlagerung von autogener Spongiosa in den Osteotomiespalt und statische Reverriegelung zur Längenund Torsionssicherung. Zeitgerechte Ausheilung mit regelrechten Längen- und Torsionsverhältnissen klinisch und computertomografisch (mit freundlicher Genehmigung von Springer Science + Business Media: Strecker W, Keppler P, Schulte M. Maßnahmen bei fehlgeschlagenen Osteosynthesen am proximalen Femur. Trauma Berufskrankh 2003; 5 (Suppl. 2): 184-191).
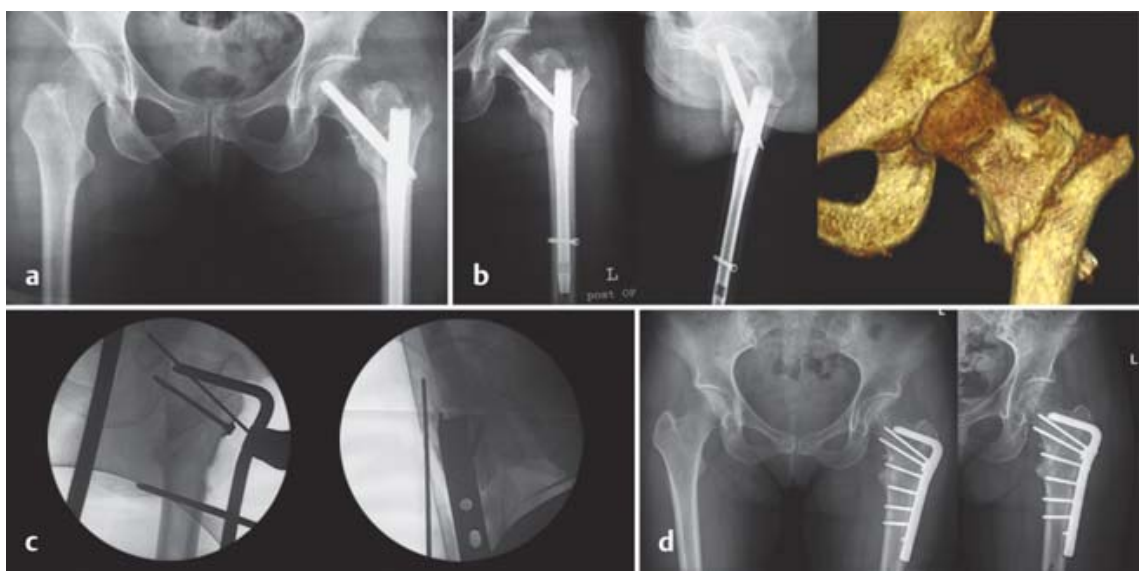

Abb.9 a bis d 50-jährige Patientin mit a Osteosynthese einer proximalen Femurfraktur links vom Typ AO 31-A1.1 mittels Gammanagel. b Es resultiert eine mehrdimensionale Deformität klinisch, radiografisch und computertomografisch: $15^{\circ}$ Varus; $2,2 \mathrm{~cm}$ Translation; $1,5 \mathrm{~cm}$ Verkürzung; $8^{\circ}$ Innentorsionsabweichung. c Zunächst Metallentfernung, dann entsprechend der Korrekturplanung Vorbereiten des Klingensitzes für die $95^{\circ}$-Kondylenplatte, Montage eines Femurdistraktors, proximal in der Crista iliaca, distal in der Femurdiaphyse verankert. Anschließend schräge Osteotomie in der ehemaligen Frakturzone. Nach plangerechter Korrektur unter Zuhilfenahme des Femurdistraktors und eines diaphysären Joysticks präliminare interfragmentäre Kompression durch Schraubenosteosynthese, komplettiert durch die abschließende Osteosynthese mittels der $95^{\circ}$-Kondylenplatte. d Auf dem postoperativen Röntgenbild rechts sind die Verankerungspunkte für den Femurdistraktor zu erkennen.

ren oder treppenförmigen Osteotomien [10].

\section{Horizontale Achsdeformitäten}

Translationen können sowohl die frontale als auch die sagittale Achse oder auch beide Achsen betreffen. In praxi wirken sie sich allerdings bevorzugt in der Frontalebene aus. Über eine Verschiebung der mechanischen Beinachse kommt es zu Fehlbelastungen von Knie- und Sprunggelenken. Jede Medialisierung des Femurschafts führt tendenziell zu einem Genu valgum, jede diaphysäre Lateralisierung zu einem Genu varum. Das Ausmaß dieser valgischen oder varischen Situation, primär das Kniegelenk betreffend, modifiziert schließlich die Indikation zur Korrekturosteotomie. Unabhängig davon beeinflussen intertrochantäre Translationen die muskuloligamentäre Balance pelvitrochantär.

\section{Längendifferenzen}

Bei Längendifferenzen der Oberschenkel ist durch eine subtile Analyse der Beingeometrie eindeutig zu klären, ob diese Deformitäten diaphysär oder intertrochantär angesiedelt sind. Längendifferenzen proximal des Trochanter minor sind extrem selten und lassen sich meist nur durch Änderung des CCD-Winkels beeinflussen. Alle sonstigen Längendifferenzen sind letztlich subtrochantär bzw. diaphysär auszugleichen (Abb.7). Hierbei können im Einzelfall akute Verlängerungen bis zu $3 \mathrm{~cm}$ durch eine treppenförmige subtrochantäre Verlängerungsosteotomie realisiert werden [10]. Limitierender Faktor sind hier zum einen die Weichteilspannung, zum anderen drohende Dehnungsschäden insbesondere des N. ischiadicus. Alternativ empfehlen sich kontinuierliche Verlängerungstechniken, etwa mittels unilateralem Distraktionsfixateur über liegendem Marknagel (Abb. 7).

\section{Torsionsabweichungen}

Coxa valga antetorta und Coxa vara retrotorta gehen naturgemäß mit unphysiologischen femoralen Torsionen einher. Posttraumatische Torsionsdifferenzen intertrochantär sind relativ selten, treten jedoch subtrochantär und proximal diaphysär insbesondere nach Osteosynthesen durch proximale Femurnägel durchaus häufiger auf.

Torsionskorrekturen sind nur angezeigt bei fehlendem rotatorischem Nulldurchgang sowohl im extendierten als auch im flektierten Hüftgelenk [11]. Dementsprechend definiert sich das Korrekturziel: Bei posttraumatischen Veränderungen ist die femorale Torsion der gesunden unverletzten Gegenseite anzustreben (Abb. 8). Bei kongenitalen Veränderungen und bilateralen posttraumatischen Torsionsdeformitäten ist der freie rotatorische Nulldurchgang im Hüftgelenk das Ziel korrigierender operativer Bemühungen (Abb. 7 und 8).

Die intraoperative Torsionskontrolle erfolgt, wie oben angegeben, durch Eindrehen von je 1 Schanz-Schraube proximal und distal der geplanten Osteotomie 


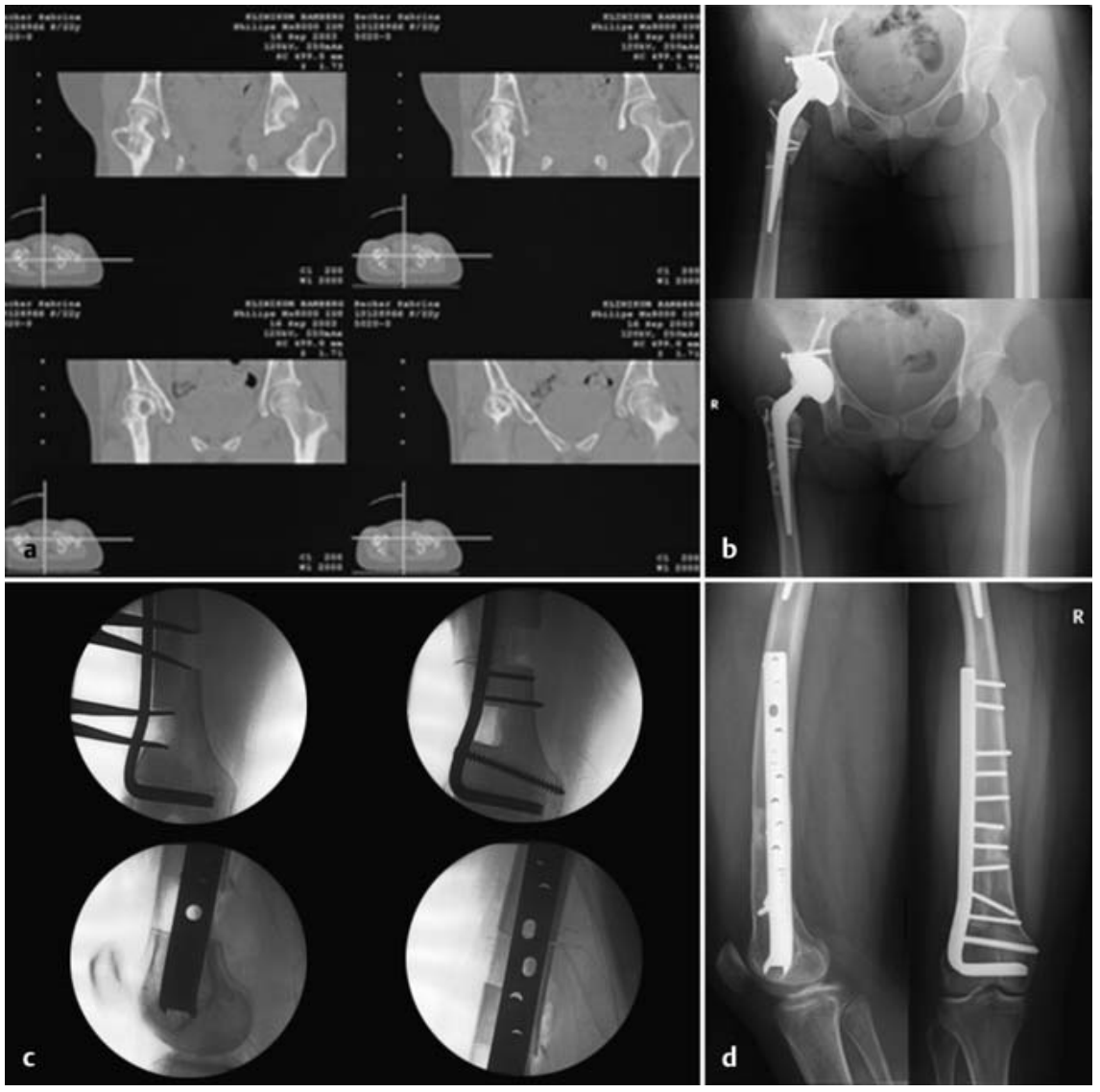

Abb. 10a bis d 22-jährige Patientin mit a koxofemoraler Destruktion rechts nach Resektion einer Neuroblastommetastase im rechten Schenkelhals im 4. Lebensjahr, nachfolgender Radiatio und Chemotherapie sowie einer intertrochantären Valgisationsosteotomie im 7. Lebensjahr. b Autogene Pfannendachplastik, subtrochantäre Varisationsosteotomie und Implantation einer zementfreien Hüft-TEP mit Keramik-Keramik-Gleitpaarung und torsionsneutralem Konusschaft. Die präoperative Verkürzung des rechten Beines von $6,5 \mathrm{~cm}$ kann dadurch bis auf $3 \mathrm{~cm}$ reduziert werden. c Definitiver Längenausgleich durch suprakondyläre treppenförmige einzeitige Verlängerungsosteotomie mit Auffüllung der Halbschaftdefekte durch allogene Knocheninterposition. d Hier abgeschlossene knöcherne Heilung (mit freundlicher Genehmigung von Springer Science + Business Media: Strecker W, Suger G, Schulte M. Das destruierte Hüftgelenk. Trauma Berufskrankh 2010; 12 (Suppl. 2): 132-141).
(Abb. 8). Diese Schanz-Schrauben dienen als Zeiger für die Torsion und dürfen nicht als Hebel, sprich Joystick, missbraucht werden!

Proximale Femurosteotomien werden nach wie vor bevorzugt mit Winkelplatten stabilisiert. Den weitesten Anwendungsbereich bietet hierbei die $95^{\circ}$-Kondylenplatte. Bei Valgisierungen empfehlen sich darüber hinaus $120^{\circ}$ oder $130^{\circ}$-Winkelplatten, bei Varisierungen gekröpfte Rechtwinkelplatten [4].

Als Zugang wird der laterale Zugang zum Femur genutzt. Ist ein flächiger Kontakt der knöchernen Fragmente in der Osteotomieebene gegeben, empfehlen wir eine postoperative Teilbelastung von $20 \mathrm{~kg}$ über einen Zeitraum von 6 Wochen bei freifunktioneller Nachbehandlung. Der anschließende Belastungsaufbau erfolgt mit $10 \mathrm{~kg}$ pro Woche. Nach Drehosteotomien des Schenkelhalses orientieren sich Belastungsaufbau und Mobilisierung an den Richtlinien wie nach Schraubenosteosynthesen von Schenkelhalsfrakturen.

Gelegentlich lassen sich posttraumatische Deformitäten elegant über liegende Implantate ausgleichen. Dieses bietet sich insbesondere für Torsionskorrekturen nach vorangegangener Osteosynthese mit Marknagel und Schenkelhalsschraube an (Abb. 8).

Die seltene Kombination posttraumatische Deformität und Pseudarthrose kann prinzipiell akut korrigiert werden, wenn Ort der Fehlstellung und Pseudarthrose
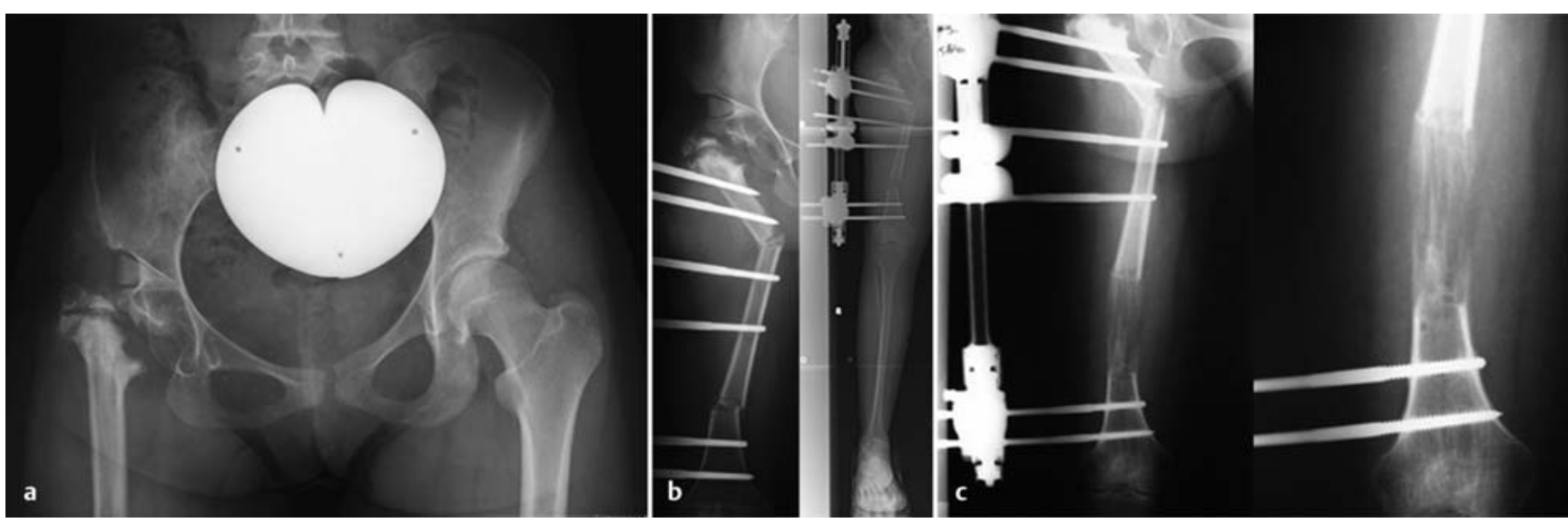

Abb. 11 a bis c 15-jährige Patientin mit a Girdlestone-Situation rechts nach Säuglingskoxitis und späterer frustraner Azetabuloplastik. Aktuell Hypotrophie und Verkürzung des rechten Beines um $8 \mathrm{~cm}$. b Pelvic-Support-Osteotomie durch minimalinvasive Bohrloch-Meißel-Osteoklasie am Übergang vom proximalen zum mittleren Femurviertel mit akuter Valgisation und Extension sowie analoge Osteoklasie am Übergang vom 3. zum 4. Femurviertel zur anschließenden kontinuierlichen Varisation und Verlängerung. c Zeitgerechte Kallusreifung im Bereich der proximalen und distalen Osteoklasie (mit freundlicher Genehmigung von Springer Science + Business Media: Strecker W, Suger G, Schulte M. Das destruierte Hüftgelenk. Trauma Berufskrankh 2010; 12 (Suppl. 2): 132-141). 
identisch sind. Alternativ, insbesondere bei zusätzlichen posttraumatischen Längenverlusten, sind kombinierte Verfahren zu erwägen, etwa mit akuter Korrektur der Deformität (frontal, sagittal, horizontal; Torsion) und anschließender kontinuierlicher Kompression der Pseudarthrose und gleichzeitigem Längengewinn (Abb. 7).

\section{Koxofemorale Destruktion}

Wie in der Einleitung bereits erwähnt, können besonders in der Wachstumsphase erhebliche Deformitäten und Zerstörungen koxofemoral auftreten, häufig begleitet von einer Hypotrophie von betroffener Beckenhälfte und unterer Extremität, meist Knochen und umgebende Weichteile mit einschließend. Ursache können frühkindliche Traumata sein, Tumorresektionen oder entsprechende tumorassoziierte Therapien wie lokale Rekonstruktionen und Bestrahlung (Abb. 10). Destruktionen des Hüftgelenks nach nicht rechtzeitig therapierter bakterieller Säuglingskoxitis sind in industrialisierten Ländern extrem selten und kommen häufiger im tropischen Kontext vor. Die Folgen für die betroffenen Patienten sind gravierend, auch später scheiden meist alloarthroplastische Maßnahmen am Hüftgelenk aus. Hier kann im Einzelfall die Pelvic-Support-Osteotomie segensreich sein. Es handelt sich hierbei um eine Weiterentwicklung der Schanz-Osteotomie durch die Schule von G.A. Ilizarov [2]. Hierbei wird eine akute Valgisations-Extensions-Osteotomie im proximalen Viertel des Femurs durchgeführt in Kombination mit einer kontinuierlichen Varisation und Verlängerung durch eine distale Femurosteotomie [7]. Die funktionellen Ergebnisse sind hierbei überraschend gut. Letztlich kommt es zur Ausbildung eines pelvifemoralen Walzennearthros (Abb. 11).

\section{Literatur}

${ }^{1}$ Ganz R, Leunig M, Leunig-Ganz Ket al. The etiology of osteoarthritis of the hip: an integrated mechanical concept. Clin Orthop Relat Res 2008; 466: 264-272

2 Ilizarov GA. Transosseus Synthesis. Berlin, Heidelberg, New York, Tokyo: Springer; 1992

${ }^{3}$ Kempf I, Karger C, Bikhalil JA et al. L'osteótomie de retournement en arrière de la tête fémorale dans la nécrose de la tête. Rev Clin Orthop 1984; 70: 271-282

${ }^{4}$ Müller ME, Allgöwer M, Schneider R et al. Manual der Osteosynthese. Berlin, Heidelberg, New York: Springer; 1977

5 Müller ME, Nazarian S, Koch P et al. The comprehensive Classification of Fractures of long Bones. Berlin, Heidelberg, New York, Tokyo: Springer; 1990

${ }^{6}$ Pauwels F. Atlas zur Biomechanik der gesunden und kranken Hüfte. Berlin, Heidelberg, New York: Springer; 1973
${ }^{7}$ Rozbruch RS, Paley D, Bhave A et al. Ilisarov hip reconstruction for the late sequelae of infantile hip infection. J Bone Joint Surg [Am] 2005; 87: 1007-1018

${ }^{8}$ Strecker W, Keppler P, Gebhard F et al. Length and torsion of the lower limb. J Bone Joint Surg [Br] 1997; 79: 1019-1023

${ }^{9}$ Strecker W, Keppler P, Kinzl L et al. Proximale Femurosteotomien zur Korrektur posttraumatischer Veränderungen. Chirurg 1998; 69: 1153-1160

10 Strecker W, Keppler P, Kinzl L. Die treppenförmige subtrochantäre Verlängerungsosteotomie des Oberschenkels. Operat Orthop Traumatol 1999; 11: 1-10

${ }^{11}$ Tönnis D, Heinecke A. Acetabular and femoral anteversion: relationship with osteoarthritis of the hip. J Bone Joint Surg [Am] 1999; 81 : 1747-1770

12 Sugioka Y, Hotokebuchi T, Tsutsui H. Transtrochanteric anterior rotational osteotomy for idiopathic and steroid induced necrosis of the femoral head: indications and long-term results. Clin Orthop Relat Res 1992; 277: $111-120$

\section{Prof. Dr. med. Wolf Strecker} Chefarzt

Klinik für Orthopädie und Unfallchirurgie

Klinikum am Bruderwald

Buger Straße 80

96049 Bamberg

chirurgie2@sozialstiftung-bamberg.de 